Lit hi u - A luminum - Tetraviny late

On 7.6 g (0.02 mole) $\mathrm{LiAlH}_{4}$ (100 mesh, crystalline) in $100 \mathrm{ml}$ of dry ether was condensed $11.4 \mathrm{~g}(0.045$ mole) of $\left(\mathrm{CH}_{2}=\mathrm{CH}\right)_{2} \mathrm{Hg}$. The reaction mixture was allowed to warm slowly to room temperature and stirred for $16 \mathrm{hrs}$ (overnight) and then filtered in an $\mathrm{N}_{2}$ atmosphere. During this time 0.0399 mole of hydrogen (calcd. 0.04 mole) was evolved, pumped off and measured. The ether was then removed in vacuo and the remaining soluble, fluffy precipitate extracted with several portions of benzene. Upon cooling a white material precipitates which is filtered off and dried in vacuum. Recrystallized from benzene, the very fine white needles decompose at $180^{\circ} \mathrm{C}$.
Anal. calcd. for $\mathrm{LiAlC}_{8} \mathrm{H}_{12}$ :

$$
\text { Li } 4.88 \quad \mathrm{Al} 18.98 \quad \text { C } 67.61 \quad \text { H } 8.51 \text {. }
$$

Found: $\mathrm{Li} 4.90$ Al 19.30 C 67.30 H 8.20.

The material is pyrophoric and burns in air with a sparkling flame.

In addition to infrared spectra, elemental analysis and molecular weight determinations, structural proof for the vinylaluminum compounds was provided by N.M.R. spectra taken at the Naval Ordnance Test Station, China Lake, California, for which we would like to extend our gratitude to Messrs. D. W. Moone and J. A. HAPPE ${ }^{20}$. The authors also wish to thank Dr. Charles P. Haber for his helpful suggestions during the course of this work.

20 D. W. Moore and J. A. Happe, J. physic. Chem. 65, 224 [1961].

\title{
Die dhemische Natur des Wehrsekretes von Pseudophonus pubescens und Ps. griseus
}

VIII. Mitteilung über Insektenabwehrstoffe*

Von H. Schildknecht und K. H. Weis

Aus dem Institut für Organische Chemie der Universität Erlangen

(Z. Naturforschg. 16 b, 361-363 [1961] ; eingegangen am 26. November 1960)

Bei der Käfergattung Pseudophonus konnte als Abwehrstoff Ameisensäure nachgewiesen werden. Die Käfer speichern etwa 2 mg der 70- bis 75-proz. Säure in jeder der paarig angeordneten Pygidialblasen.

\section{Allgemeines}

In Deutschland kommen lediglich zwei Vertreter der Gattung Pseudophonus vor, es sind dies Pseudophonus pubescens Müll. und Ps. griseus Panz ${ }^{1}$. Vom April bis August sind diese auf Feldern und Fluren in großer Zahl aufzufinden. Auch unter Steinen an den Rändern sandiger Feldwege wird man sie häufig antreffen. Tagsüber suchen sie unter Erdschollen und Grasnarben Schutz vor dem Licht, wobei sie stets darauf bedacht sind, einen feuchten Schlupfwinkel ausfindig zu machen.

Der Käfer wird man leicht habhaft, wenn man nach einem längeren Regen am Rande von Getreidefeldern Schollen und die beim Pflügen losgerissenen Grasnarben umwendet. Vielfach leben $P$ s. pubescens $(14-16 \mathrm{~mm})$ und Ps. griseus $(9-11 \mathrm{~mm})$ gesellschaftlich zusammen. Zur Haltung wurden Terrarien mit Ackererde gefüllt und einige flache Steine dienten als Unterschlupf. Als Futter eigneten sich kleine

* II. Mitteilung s. Z. Naturforschg., im Druck.

1 E. Reitrer, Fauna Germanica, die Käfer des Deutschen Reiches, K. G. Lutz Verlag, Stuttgart 1908, Bd. I, S. 170.
Rindfleischstückchen sowie zerstampfte Schnecken und Regenwürmer.

$$
\text { Die Pygidialblasen }
$$

Wie bei anderen Laufkäferarten befinden sich an beiden Seiten des Afters die Ausführungsgänge der Pygidialblasen. Diese sind hell und durchsichtig, ihre Wand ist von Muskelfasern durchzogen, welche eine Kontraktion des Reservoirs und damit ein Entleeren desselben ermöglichen. Die zur Längsachse des Käfers symmetrisch angeordneten beiden Blasen erreichen eine Länge von $1,5-2 \mathrm{~mm}$, sobald sie mit Sekret prall angefüllt sind. Das Sekret selbst stellt eine wasserhelle, relativ flüchtige Flüssigkeit dar. Bei Bedrohung durch einen Feind wird es bis zu einer Entfernung von etwa $20 \mathrm{~cm}$ in feinen Tröpfchen gezielt ausgespritzt.

\section{Analyse des Abwehrstoffes}

$$
\text { Vorproben }
$$

Der Geruch des Abwehrstoffes ist scharf und stechend. Die Nasenschleimhaut erleidet eine Verätzung 
und schon aus diesem Grunde ist es nicht mehr möglich, eine präzisere Beschreibung zu geben. Säure-BasenIndikatoren zeigen eine stark saure Reaktion und machen, im Verein mit der die Haut reizenden Eigenschaft, das Vorliegen einer Säure wahrscheinlich.

Für den papierchromatographischen Nachweis und die spektralphotometrische, quantitative Bestimmung der mutmaßlichen Säure war eine Lösung in optisch reinem Alkohol, für den Versuch der Mikrobestimmung eine wäßrige Lösung vonnöten. Die beiden Lösungsmittel befanden sich in der Vorlage einer Absaugvorrichtung ${ }^{2}$, welche beim Vorliegen flüchtiger Sekrete stets ein schnelles und zuverlässiges Arbeiten gestattet.

\section{Papierchromatographie des Sekrets}

Da irgendwelche Hinweise auf die Art der im Pseudophonussekret vorhandenen Säure nicht vorlagen, mußte zur papierchromatographischen Untersuchung ein möglichst universelles Lösungsmittelsystem herangezogen werden. Long und Mitarbb. ${ }^{3}$ konnten mit einem Fließmittelgemisch aus Äthanol : Wasser : Ammoniak (80:16:4) verschiedene Säuretypen, bei guter Trennung homologer Säuren, nebeneinander nachweisen. Verwendung dieses Gemisches als mobile Phase ergab im vorliegenden Falle nur einen Fleck für den Blaseninhaltsstoff beider Pseudophonusarten. Zum Sichtbarmachen des Fleckes diente eine Ninhydrinlösung. Vergleichsweise mitaufgetragene Ameisensäure gab, im Gegensatz zu verschiedenen anderen Fettsäuren, sowohl in Farbe als auch im $R_{f}$-Wert von $0,48 \mathrm{mit}$ dem Sekret vollkommene Übereinstimmung.

\section{Mikrochemischer Nachweis von A meisen s äure}

Zur Bekräftigung des papierchromatographischen Befunds über das Vorliegen von Ameisensäure in den Pygidialblasen der beiden Pseudophonusarten sollte ein weiterer, absolut zuverlässiger Nachweis treten. Hierzu bot sich eine von FrehDen und Fürst ${ }^{4}$ vorgeschlagene Mikromethode an, die genügend selektiv und spezifisch ist. Sie beruht auf der Fähigkeit der Ameisensäure, elementares Brom zu reduzieren. Das bei der Oxydation der Säure entstehende Kohlendioxyd läßt sich in einfacher Weise durch Einleiten in Bariumhydroxydlösung und Fällung als Bariumcarbonat nachweisen. Bei unseren Experimenten gelangte eine wäßrige Sekretlösung zur Anwendung, welche je nach der Zahl der abgesaugten ${ }^{2}$ Käfer im allgemeinen 1 bis $2 \mathrm{mg} / \mathrm{cm}^{3}$ Ameisensäure enthielt. Diese Mengen bewirkten eine reichliche Fällung von Bariumcarbonat und führten dadurch zu einer eindeutigen Identifizierung der Pygidialblasen-Sekrete von Pseudophonus pubescens und Ps. griseus.

Bestimmung der je Käfer sezernierten A meisens äuremenge

Zur quantitativen Bestimmung der Ameisensäure, wurde die äthanolische Lösung spektroskopiert (Abb. 1). Dabei enthielt die Sekretlösung die von fünf Käfern abgegebene Ameisensäure in $4 \mathrm{ml}$ Äthanol gelöst.

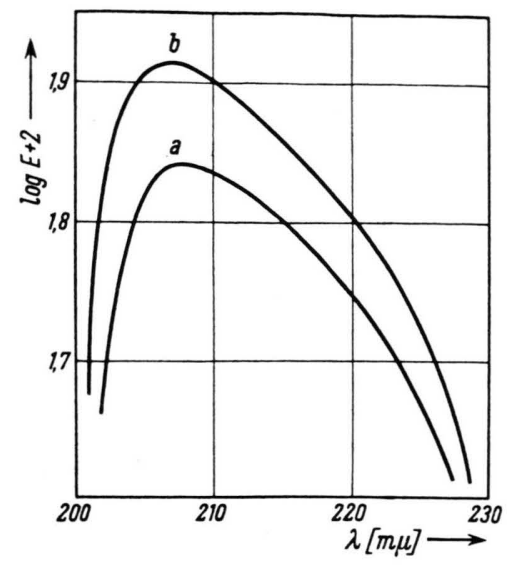

Abb. 1. UV-Absorptions-Spektren von $8 \mathrm{mg}$ Ameisensäure in $4 \mathrm{ml}$ Äthanol (a) und des Sekrets von 5 Pseudophonus pubescens in $4 \mathrm{ml}$ Äthanol (b).

Durch einen Vergleich der in Abb. 1 dargestellten Absorptionskurven errechnet sich, daß in den Pygidialdrüsen-Säcken eines Pseudophonus pubescens durchschnittlich etwa $1,9 \mathrm{mg}$ Ameisensäure vorhanden sein müssen. Ganz analog erfolgte die Bestimmung der sezernierten Menge an Ameisensäure beim Ps. griseus, wobei sich ein durchschnittlicher Gehalt von etwa $1,6 \mathrm{mg}$ Ameisensäure je Blasenpaar ergab.

Dieser Wert konnte durch eine Säuretitration des aus den Blasen direkt gewonnenen Sekrets bestätigt werden. Dazu wurden jeweils 10 Käfer in einem Becherglas mittels eines mit Essigester getränkten Wattebausches getötet und die beiden Blasen operativ entfernt. Nach dem Abreißen von Flügeldecken und Flügeln konnten die beiden letzten dorsalen Hinterleibssegmente mit einer Schere abgetrennt und beiseite gelegt werden. Neben anderen inneren Organen waren die Reservoirs an ihrer eiförmigen Gestalt und Durchsichtigkeit leicht $\mathrm{zu}$ erkennen und mit einer scharfen Rasierklinge vom ventralen Segment zu entfernen. Die so erhaltenen, prall gefüllten Blasen wurden in einem kleinen Erlenmeyerkölbchen mit einem Glasstab unter $10 \mathrm{ml}$ dest. Wasser zerdrückt, so daß sich ihr Inhalt mit dem Wasser vermischen konnte. Nach Zusatz eines Tropfens Phenolphthalein-Lösung ergab die Titration mit $n / 10$ Natronlauge einen Verbrauch von $4,4 \mathrm{ml}$ für Pseudophonus pubescens und $4,0 \mathrm{ml}$ für Ps. griseus. Das entspricht dem Vorhandensein von 2,0 mg bzw. $1,8 \mathrm{mg}$ Ameisensäure je Blasenpaar.

Zur Konzentrationsbestimmung wurden jeweils 20 Blasen herausoperiert, gewogen, zwischen Filtrierpapier zerquetscht und das Gewicht der verbleibenden Häute bestimmt. Die folgende Tabelle zeigt die Ergebnisse:

2 H. Schildknecht, Angew. Chem. 71, 524 [1959].

3 A. G. Long, J. R. Quayle u. R. J. Stedman, J. chem. Soc. [London] 1951, 2197.

4 O. Frehden u. K. Fürst, Mikrochemie 25, 256 [1938]. 


\begin{tabular}{|l|c|c|}
\hline & $\begin{array}{c}\text { Ps. pub. } \\
{[\mathrm{mg}]}\end{array}$ & $\begin{array}{c}\text { Ps. gris. } \\
{[\mathrm{mg}]}\end{array}$ \\
\hline $\begin{array}{c}\text { Gewicht von 20 gefüllten } \\
\text { Blasen }\end{array}$ & 38,4 & 31,6 \\
$\begin{array}{c}\text { Gewicht von 20 geleerten } \\
\text { Blasen }\end{array}$ & 10,0 & 7,2 \\
$\begin{array}{c}\text { Inhalt von 20 Pygidial- } \\
\text { blasen }\end{array}$ & 28,4 & 24,4 \\
\hline
\end{tabular}

Tab. 1. Gehalt der Pygidialblasen an Ameisensäure.

Zusammen mit dem Befund über das Vorliegen von $20 \mathrm{mg}$ bzw. $18 \mathrm{mg}$ reiner Ameisensäure in je 20 Blasen von Pseudophonus pubescens bzw. Ps. griseus ergab sich ein Gehalt von 71 bzw. 74 Prozent. Dieses Ergebnis ist keinesfalls absolut zu werten, denn kleinere Fehler sind bei der Versuchsweise nicht auszuschließen und zudem handelt es sich um einen Durchschnittswert.

\section{Diskussion}

Daß das Versprühen von Ameisensäure durch die Ps.-Arten ihrer Verteidigung dient, kann kaum an- gezweifelt werden. Auf jede Art von Reiz hin, sei es Zwicken, Drücken, Wärme oder Mangel an Atemluft, entleeren die Käfer zunächst eine Blase in Richtung auf den Angreifer hin und wenn diese geleert ist, auch die zweite. Die fein zerstäubte Ameisensäure benetzt die nächste Umgebung des Käfers nahezu vollkommen.

Die Gattung Pseudophonus wird dem Tribus der Harpalinae zugeordnet und gehört damit zur Familie der Laufkäfer oder Carabiden ${ }^{1}$. Daher überrascht es, daß das Produkt ihrer Pygidialdrüsen seiner chemischen Natur nach nicht mit dem der meisten Laufkäferarten übereinstimmt. Immerhin handelt es sich hierbei noch um eine Säure, während bei den Brachynini Chinone als Abwehrstoffe auftreten $^{5}$.

Der Deutschen Forschungsgemeinschaft danken wir für die Unterstützung dieser Untersuchungen.

5 H. Schildknecht u. K. Holoubek, Angew. Chem. 73, 1 [1961].

\title{
Reinigung und Charakterisierung des an T2-Phagen gebundenen Lysozyms
}

\author{
Von W. Katz und W. WeIDEL \\ (Z. Naturforschg. 16 b, 363-368 [1961] ; eingegangen am 28. März 1961)
}

\begin{abstract}
Procedures for the isolation, and some properties of the particle-bound T2 phage lysozyme are described. The enzyme appears to have a higher molecular weight $(21000)$ and a correspondingly lower specific activity than the homologous free lysozyme, which otherwise it resembles most closely.
\end{abstract}

In einer vorhergehenden Arbeit ${ }^{1}$ wurde über die Isolierung des lytischen Enzyms berichtet, welches in Escherichia coli B-Zellen nach Infektion mit T2Phagen auftritt. Dieses Enzym unterbricht die intrazelluläre Phagenvermehrung durch Lyse der Bakterienzelle, wobei es zusammen mit den neugebildeten Phagenteilchen ins Lysat abgegeben wird ${ }^{2,3}$. Hieraus kann es ohne weiteres isoliert werden.

Während dieses Enzym nicht an irgendwelche

1 W. Weidel u. W. Katz, Z. Naturforschg. 16 b, 156 [1961].

2 G. Koch u. E. Jordan, Biochim. biophysica Acta [Amsterdam] 25, 437 [1957].

3 G. Koch u. W. Dreyer, Virology 6, 291 [1958].

4 W. Weidel u. J. Primosigh, Z. Naturforschg. 12 b, 421 [1957].

5 W. WEIDEL, Z. Naturforschg. 6 b, 251 [1951].

6 W. WEIDEL, Z. Naturforschg. 7 b, 145 [1952].

7 G. Koch u. W. W EIDEL, Z. Naturforschg. 11 b, 345. [1956].

8 W. Weidel u. J. Primosigh, J. gen. Microbiol. 18, 513 [1958].
Phagenstrukturen gebunden, sondern in freier Form vorliegt, läßt sich andererseits aus gereinigten, hochtitrigen T2-Suspensionen auf einfache Weise ein an Phagenteilchen gebundenes Enzym gewinnen ${ }^{4}$, dessen zerstörende Wirkung auf E. coli B-Zellwände erstmalig von WeIDEL ${ }^{5}$ beschrieben wurde.

Dieses teilchengebundene Enzym, bereits in mehrfacher Hinsicht untersucht ${ }^{6-14}$, spielt vermutlich eine entscheidende Rolle bei der Ingangsetzung des T2-

9 W. Weidel, H. Frank u. H. H. Martin, J. gen. Microbiol. 22, 158 [1960].

10 J. Primosigh, H. Pelzer, D. Maass u. W. Weidel, Biochim. biophysica Acta [Amsterdam] 46, 68 [1960].

11 L. F. Barrington u. L. M. Kozloff, Science [Washington] 120, 110 [1954].

12 D. D. Brown u. L. M. Kozloff, J. biol. Chemistry 225, 1 [1957].

13 L. M. Kozloff u. M. Lute, J. biol. Chemistry 228, 529 [19.57].

14 L. M. Kozloff u. M. Lute, J. biol. Chemistry 234, 539 [1959]. 